\title{
EDITORIAL DEVICES
}

\begin{tabular}{|c|c|c|}
\hline$a b c$ & italic & single underline \\
\hline $\mathrm{ABC}$ & small capitals & DOUBLE UNDERLINE \\
\hline $\mathrm{ABC}$ & roman capitals & TRIPLE UNDERLINE \\
\hline$A B C$ & italic capitals & QUADRUPLE UNDERLINE \\
\hline$A B C$ & italic capitals bold & FIVE OR MORE UNDERLINES \\
\hline $\mathrm{abc}$ & bold & words emphasized but not underlined \\
\hline$a b e$ & strikethrough & deleted words or letters \\
\hline$\sim a b c \sim$ & curved line & $\sim$ words indicated by ditto marks $\sim$ \\
\hline$\{a b c\}$ & curved brackets & $\{$ words inserted above, below, or \\
\hline & & beside the line\} \\
\hline$[\mathrm{abc}]$ & square brackets & [material provided by editor $]$ \\
\hline$\wedge a b c^{\wedge}$ & carets & $\wedge_{\text {words written upside down }}{ }^{\wedge}$ \\
\hline$>$ & angle brackets & used to enclose $<$ over $>$ at bottom of page \\
\hline & diamond & page break \\
\hline
\end{tabular}


